\title{
Rare Diseases with Periodontal Manifestations
}

\author{
Marcel Hanisch ${ }^{1,4, *}$, Thomas Hoffmann ${ }^{2}$, Lauren Bohner ${ }^{1}$, Lale Hanisch ${ }^{3}$, Korbinian Benz ${ }^{4}(\mathbb{D}$, \\ Johannes Kleinheinz ${ }^{1}$ and Jochen Jackowski ${ }^{4}$ \\ 1 Department of Cranio-Maxillofacial Surgery, Research Unit Rare Diseases with Orofacial, \\ Manifestations (RDOM), University Hospital Münster, Albert-Schweitzer-Campus 1, Building W 30, \\ D-48149 Münster, Germany; lauren@usp.br (L.B.); johannes.kleinheinz@ukmuenster.de (J.K.) \\ 2 Department of Periodontology, University Hospital Carl Gustav Carus, TU Dresden, Fetscherstraße 74, \\ 01307 Dresden, Germany; thomas.hoffmann@uniklinikum-dresden.de \\ 3 Department of Orthodontics, School of Dentistry, Faculty of Health, Witten/Herdecke University, \\ Alfred-Herrhausen-Strasse 45, 58455 Witten, Germany; lale.hanisch@gmail.com \\ 4 Department of Oral Surgery and Dental Emergency Care, Faculty of Health, Witten/Herdecke University, \\ Alfred-Herrhausen-Strasse 45, 58455 Witten, Germany; korbinian.benz@uni-wh.de (K.B.); \\ jochen.jackowski@uni-wh.de (J.J.) \\ * Correspondence: marcel.hanisch@ukmuenster.de; Tel.: +49-(0)-251-834-7002; Fax: +49-(0)-251-834-7184
}

Received: 10 February 2019; Accepted: 5 March 2019; Published: 9 March 2019

\begin{abstract}
Background: The object of this paper was to provide an overview of rare diseases (RDs) with periodontal manifestations and allocate them to relevant categories. Methods: In ROMSE, a database for "Rare Diseases with Orofacial Involvement", all 541 entities were analyzed with respect to manifestations of periodontal relevance. Inclusion criteria were periodontally relevant changes to the oral cavity, in accordance with the 2018 version of the Classification of Periodontal and Peri-Implant Diseases and Conditions. Rare diseases were recorded, using the methodology described, and subsequently compared with the Orphanet Classification of Rare Diseases. Results: A total of 76 RDs with periodontal involvement were recorded and allocated in accordance with the Classification of Periodontal and Peri-Implant Diseases and Conditions. Of the 541 RDs analyzed as having known orofacial manifestations, almost 14 percent indicated a periodontally compromised dentition. Conclusions: Around 14 percent of RDs with an orofacial involvement showed periodontally relevant manifestations, which present not only as a result of gingivitis and periodontitis, but also gingival hyperplasia in connection with an underlying disease. Thus, dentists play an important role in therapy and early diagnoses of underlying diseases based on periodontally relevant manifestations.
\end{abstract}

Keywords: rare diseases; periodontal manifestations; oral manifestations of systemic diseases; oral manifestations; classification of periodontal and peri-implant diseases and conditions

\section{Introduction}

According to the European Union (EU) definition, a disease is classified as "rare" when fewer than one in 2000 people are affected by it. At least 30 million people in the 28 member states of the EU are affected [1]. Worldwide, between 5000 and 8000 different rare diseases (RD) are known, 80 percent of which have a genetic cause [2,3]. Around four million people in the Federal Republic of Germany are affected by RDs [2]. Since 2009, there has been an increase in public awareness of RDs in the EU after the Council of the European Union called upon member states to draw up, at the appropriate level, plans and strategies for RDs [4]. In most cases, exact epidemiological data are not available as a result of incomplete registrations of RDs in national and international databases. To improve this situation, the German Ministry of Health set up a "Nationaler Aktionsplan für Menschen mit Seltenen 
Erkrankungen" (National Plan of Action for People with Rare Diseases) [5]. In the meantime, so-called European Reference networks for rare diseases have been set up for some RDs [6].

Rare Diseases can manifest themselves in nearly all organs and, as a result, they often have systemic effects. Their development is frequently chronic, progressive, and degenerative, and they can cause disabilities that impair the quality of life as well as life expectancy [7]. Patients who are affected, and their families, often face great difficulties regarding diagnoses and treatments [8]. Doctors often lack the time and resources, with little available information, to help them diagnose RDs accurately and, in turn, treat them properly [9]. In addition, incorrect diagnoses lead to mental stress for sufferers and their families [10]. As 15 percent of all RDs can manifest themselves orofacially [11,12], dental professionals play a major role in making diagnoses and deciding on treatment in their particular fields. This also applies to changes relating to the gingiva and periodontium [13]. However, there has always been room for improvement in both diagnoses and treatments, as well as in research and the exchange of information and experience with RDs [14].

To this day, there is no index of periodontally relevant RDs, nor any list of periodontal manifestations in RDs. Therefore, the object of this paper was to provide an overview of periodontal manifestations of RDs, as well as to allocate RDs to relevant categories, analogous to the 2018 version of the Classification of Periodontal and Peri-Implant Diseases and Conditions [15-24]

\section{Methods}

\subsection{System Behind the Search Criteria}

In ROMSE, the database for "Rare Diseases with Orofacial Involvement" [25], which went online 13 July 2017, all entities recorded were analyzed with respect to manifestations of periodontal relevance, such as gingivitis, periodontitis, gingival hyperplasia, or haemorrhagic diatheses. The database is based on the systematic literature research on orofacial manifestations, which has been in operation since 2011. To detect orofacial manifestations in rare diseases, in November 2011, work commenced on inspecting and evaluating rare disease databases such as Orphanet, (Online Mendelian Inheritance in Man (OMIM) (http://www.omim.org), and PubMed (https://www.ncbi.nlm.nih.gov/pubmed). The criterion for analysis of a rare disease and its orofacial manifestations was inclusion in the Orphanet Classification of Rare Diseases. This classification defines a 'rare' disease using the same definition as the EU. Thus, only rare diseases that are defined as rare in the EU were covered. The requirement of inclusion was the description of at least one dental, oral, maxillofacial, periodontal, or orthodontic symptom listed in the Orphanet, OMIM, or Pubmed databases. Medical observations in Orphanet and OMIM were analyzed for the presence of any orofacial manifestation in the respective rare disease. Our relevant literature search in Pubmed was obtained using the following enhanced keywords: "disease name" AND "cleft palate" OR "dental" OR "dysgnathia" OR "gingivitis" OR "gingival enlargement" OR "gingival hyperplasia" OR "gum bleeding" OR "hypodontia" OR "micrognathia" OR "mucositis" OR "oral health" OR "oral lesions" OR "oral and maxillofacial surgery" OR "orthodontic" OR "periodontology" OR "periodontitis" OR "prognathism" OR "stomatognathic system" OR "tooth loss" [26]. At the time of this study, 541 RDs with orofacial manifestations were listed in the database.

\subsection{Criteria for Inclusion and Exclusion}

Inclusion criteria for this publication were all periodontally relevant changes in the oral cavity, in accordance with the 2018 version of the Classification of Periodontal and Peri-Implant Diseases and Conditions [15-24]. The tongue, buccal mucosa, lips, roof of the mouth, enamel, and dentin were all disregarded. Thus, the periodontium includes the following:

- Gingiva, which surrounds the tooth and parts of the alveolar bone at the level of the neck of the tooth.

- Alveolar bone, which is part of the jaw bone in which the teeth are set.

- $\quad$ Root cementum, which covers the root surface of the teeth and parts of the apical root canal wall. 
- Periodontal fibre, which are bundles of collagen and (partly) oxytalan fibres that reach into the root element on one side, and into the alveolar inner bone tissue on the other side.

All of the RDs recorded using the methodology described were subsequently compared again with the Orphanet Classification of Rare Diseases [27]. Only those diseases which, in line with the Orphanet Classification, were listed as RDs in accordance with the EU definition continued to be included in the list. All of the diseases were discussed among the authors on the basis of the literature available on PubMed, with a view of any periodontally relevant manifestations [28], and allocated in accordance with the 2018 version of the Classification of Periodontal and Peri-Implant Diseases and Conditions [15-24]. If no consensus was reached, the decision was made by a periodontological expert (T.H.), who is professor for "Periodontology". We discussed together whether diseases that occur with insulin-resistant diabetes should automatically be accepted as "periodontally relevant". We decided against it if the actual disease does not cause periodontally relevant symptoms. Thus, only diseases that are clearly associated with periodontal relevance were included.

The ethical approval for this study was obtained from the ethical review committee (Ref. no. 2017-443-f-N), Ethikkommission der Ärztekammer Westfalen-Lippe und der Westfälischen Wilhelms-Universität, Münster, Germany.

\section{Results}

A total of 76 RDs with periodontal involvement were recorded. Accordingly, of the 541 RDs analyzed as having known orofacial manifestations, 13.86 percent showed periodontally compromised dentition. The relation between the RDs and periodontal manifestations, such as possible pathological mechanisms related to the tissue alterations or affected gene and location of the disease are described in Table 1.

Table 1 summarizes 76 RDs with pathological changes in the periodontal region. Besides single entities, the specific periodontal symptoms, the pathological mechanism (if known) that is responsible for the periodontal manifestation, and the corresponding gene locus are mentioned. The list is completed by assignment of the periodontal symptoms to the 2018 version of the Classification of Periodontal and Peri-Implant Diseases and Conditions [15-24].

The pathological mechanism leading to periodontal alteration in the case of RD is marked as "unknown" in Table 1 when it is described as "unknown" or "not researched" in the literature. In 1 RD, the pathological mechanism is characterized as "not reported" according to the literature. For 6 other RDs, there are no indications in the literature regarding the pathomechanism, and in 2 RDs, the exact pathomechanism is unknown. In the case of $10 \mathrm{RDs}$, periodontal changes are considered as "not genetically determined". In $1 \mathrm{RD}$, the gene causing the periodontal symptoms is not known.

In 21 RDs, clinical symptoms are described as "periodontitis"; in 16 RDs, as "gum bleeding, bleeding diathesis"; in 13 RDs, as "gingival enlargement"; in 7 RDs, as "gingival enlargement, fibromatosis"; in 5 RDs, as "gingivitis, periodontitis"; in 3 RDs, as "gingival enlargement, gum bleeding"; in 3 other RDs, as "gingivitis"; and in 2 RDs, as "periodontitis, gingival enlargement". According to the literature, $1 \mathrm{RD}$ each manifests as "mucosal ulcers"; "periodontal breakdown"; compromised periodontal attachment; "periodontitis, gum bleeding, bleeding diathesis"; "gingival hyperkeratosis"; "gingival candidosis"; or "gray to gray-black staining of gingiva".

Some of the pathological mechanisms leading to periodontal involvement can be summarized in particular categories. In 17 of the RDs listed in Table 1, the periodontal alteration is related to a blood-clotting disorder. In 14 RDs, changes in the immune system are held responsible as cause for pathological processes in the periodontal region; in $3 \mathrm{RDs}$, periodontal alterations are attributed to disorders of vitamins, minerals, and trace elements metabolism; and 2 RDs are blistering disorders.

After allocation of the diseases in accordance with the Classification of Periodontal and Peri-Implant Diseases and Conditions [15-24], 39 RDs were allocated to non-plaque induced gingival diseases and conditions: genetic/developmental disorders, 15 showed manifestations that could be allocated to systemic diseases and conditions that affect the periodontal attachment apparatus: 
diseases associated with immunologic disorders, 4 RDs were allocated to non-plaque induced gingival diseases and conditions: neoplasms, and 3 to non-plaque induced gingival diseases and conditions: granulomatous inflammatory conditions (orofacial granulomatosis). Moreover, a total of 3 diseases were allocated to systemic diseases and conditions that affect the periodontal attachment apparatus: other disorders that may affect periodontal tissue. Three RDs could be allocated to non-plaque induced gingival diseases and conditions: endocrine, nutritional, and metabolic disease; a further 3 RDs could be allocated to systemic diseases and conditions that affect the periodontal attachment apparatus: metabolic and endocrine disorders; 3 other RDs could be allocated to systemic diseases and conditions that affect the periodontal attachment apparatus: diseases affecting connective tissues; another 3 RDs could be allocated to systemic diseases and conditions that affect the periodontal attachment apparatus: diseases affecting the oral mucosa and gingival tissue; and $1 \mathrm{RD}$ could be allocated to non-plaque induced gingival diseases and conditions: gingival pigmentation.

\section{Discussion}

On the basis of the findings of this study, an association between periodontal alterations and rare diseases was observed. Periodontally relevant findings were demonstrated in almost 14 percent of the RDs in which orofacial manifestations had already been diagnosed. In another paper [29], 106 RDs with periodontally relevant manifestations were identified. However, the results provided by Müller 2017 are questionable, as some of the identified RDs could not be found in the Orphanet Classification of Rare Diseases [27]. Furthermore, certain periodontal manifestations were not verifiable, or were questionable, such as a disease involving insulin resistance or diabetes. For the cri du Chat Syndrome, it was indeed found that a Tannerella forsythia periodontal pathogenic bacterial species was more prevalent [30,31]; however, no patients were found to suffer from active periodontitis. Thus, the syndrome was not considered periodontally relevant.

It is well known that the periodontal diseases may be associated with systemic conditions, such as metabolic diseases, coagulation disorders, or drug administration. [32]. Additionally, the literature shows that a great number of rare diseases will present an implication on the periodontal tissue. The etiological components for these alterations are mostly related to the pathogenesis of the disease [3335], although other factors, such as poor hygiene, may be associated with the RD [36]. In this sense, it is suggested that periodontal manifestations may be a key for the diagnosis of rare diseases.

Multisystemic diseases such as Langerhans cell histiocytosis (LCH) can simulate aggressive parodontitis. Indeed, LCH was initially incorrectly interpreted as being such a disease [37]. However, if LCH is detected and correctly classified at an early stage, systemic therapies can be immediately initiated, thereby stopping the spreading of the symptoms (Figures 1-3). Changes to the gingiva, such as gingival hyperplasias, that cannot be initially classified as being a particular disease and that might be incorrectly interpreted as being medication-induced [13], are possible initial indications of the recurrence of a severe multisystemic disease such as granulomatosis with polyangiitis (Figure 4) or leukaemia (Figure 5). Serious periodontal diseases can also, at an early stage, provide differential diagnostic indications of diseases such as Ehlers-Danlos syndrome (Figure 6), which can lead to a greatly reduced quality of life, including with respect to oral hygiene [38]. 


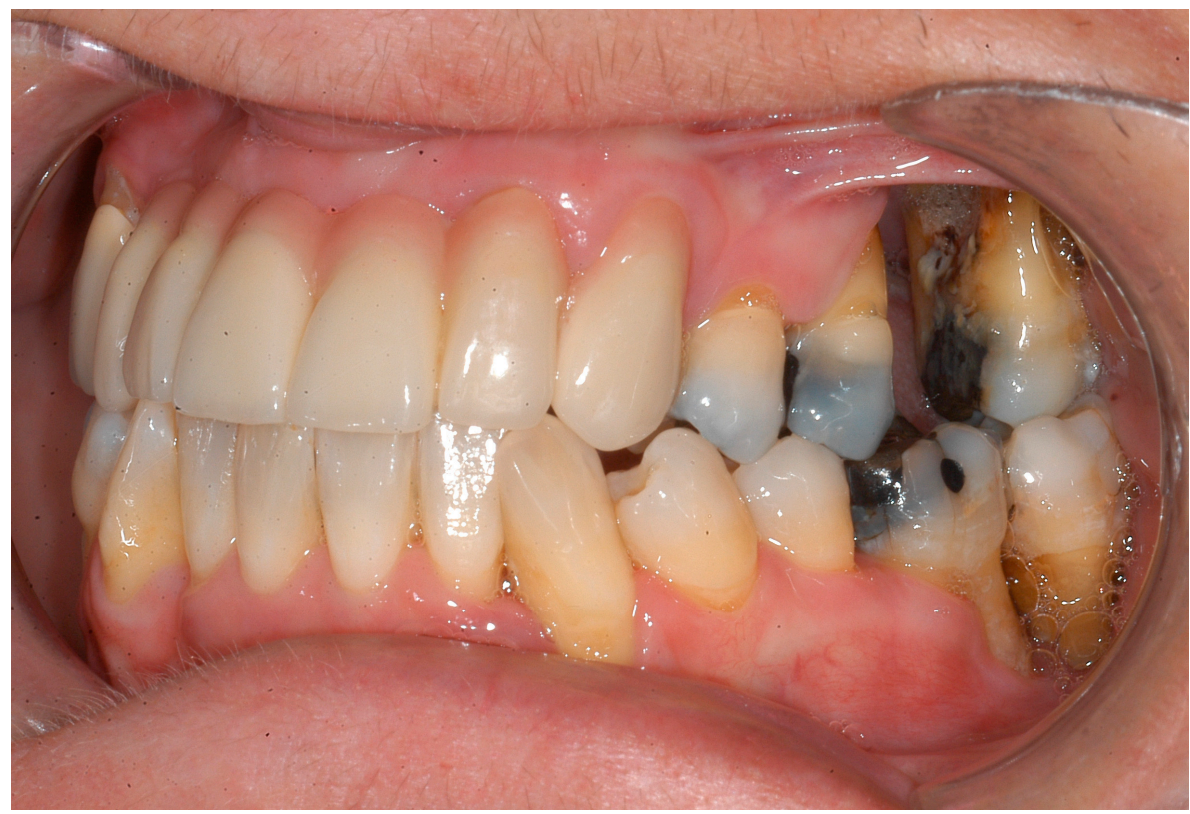

Figure 1. Excessive periodontal destruction in a 48-year-old women with Langerhans cell histiocytosis.

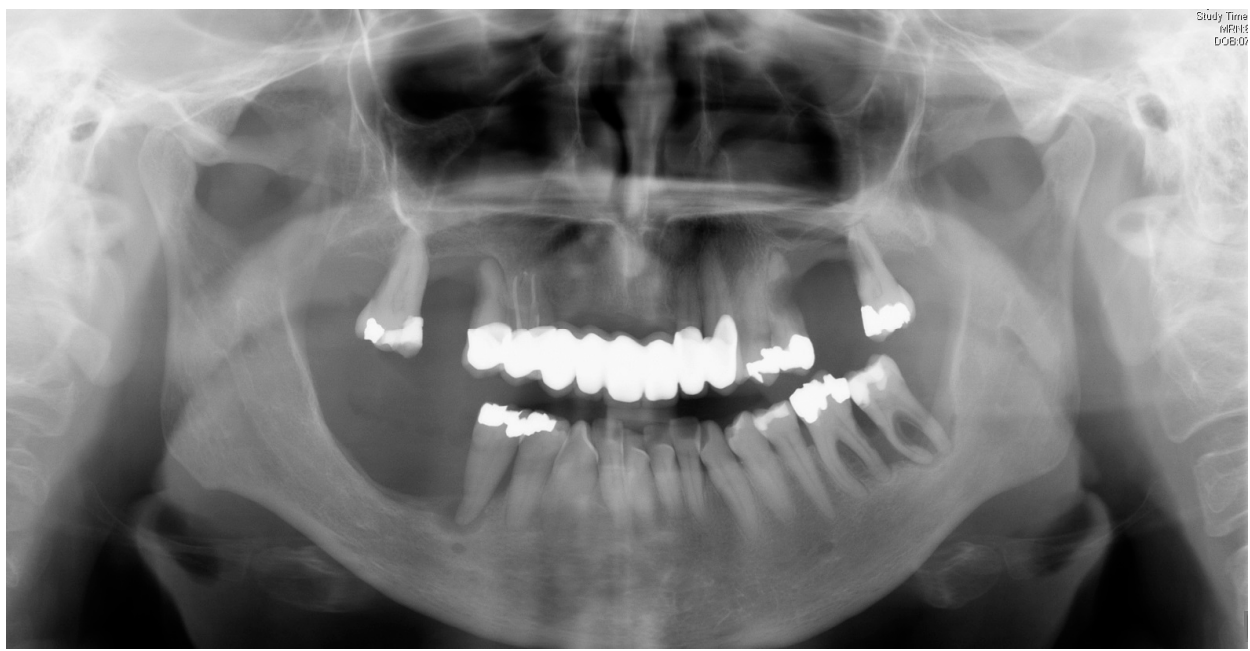

Figure 2. Excessive periodontal destruction in a 48-year-old women with Langerhans cell histiocytosis.

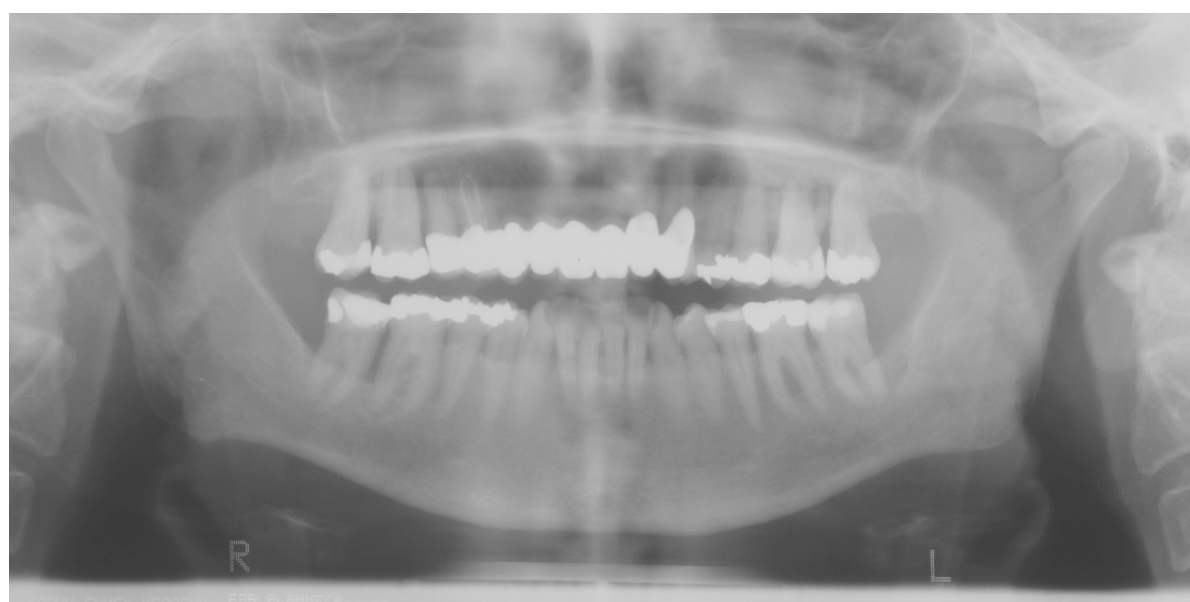

Figure 3. Same patient 12 months before: periodontal destruction was misdiagnosed and treated as aggressive periodontits. 


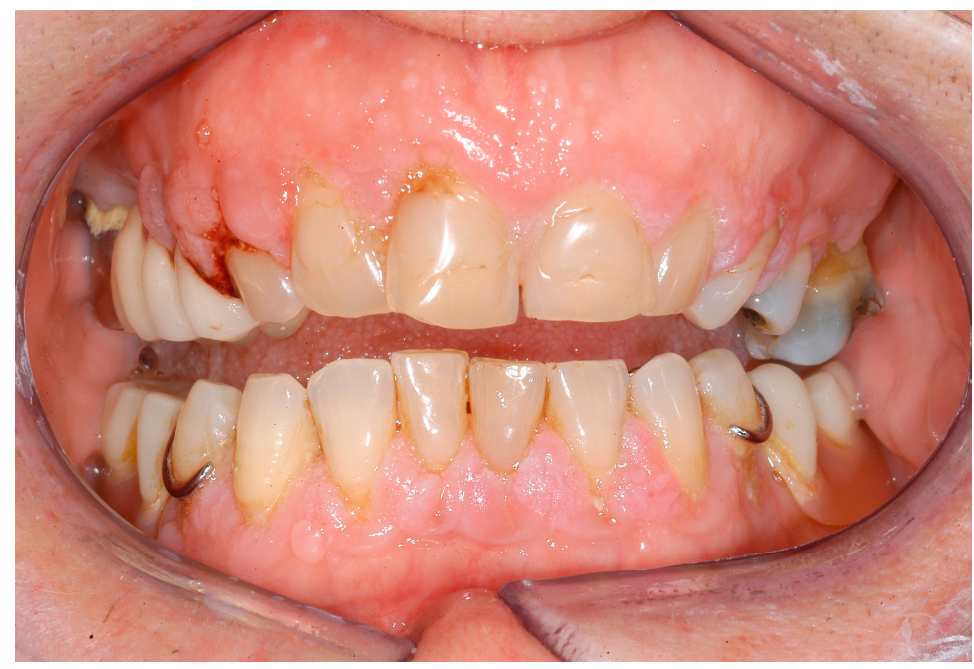

Figure 4. Gingival enlargement as a first sign of Granulomatosis with Polyangiitis in a 72-year-old women.

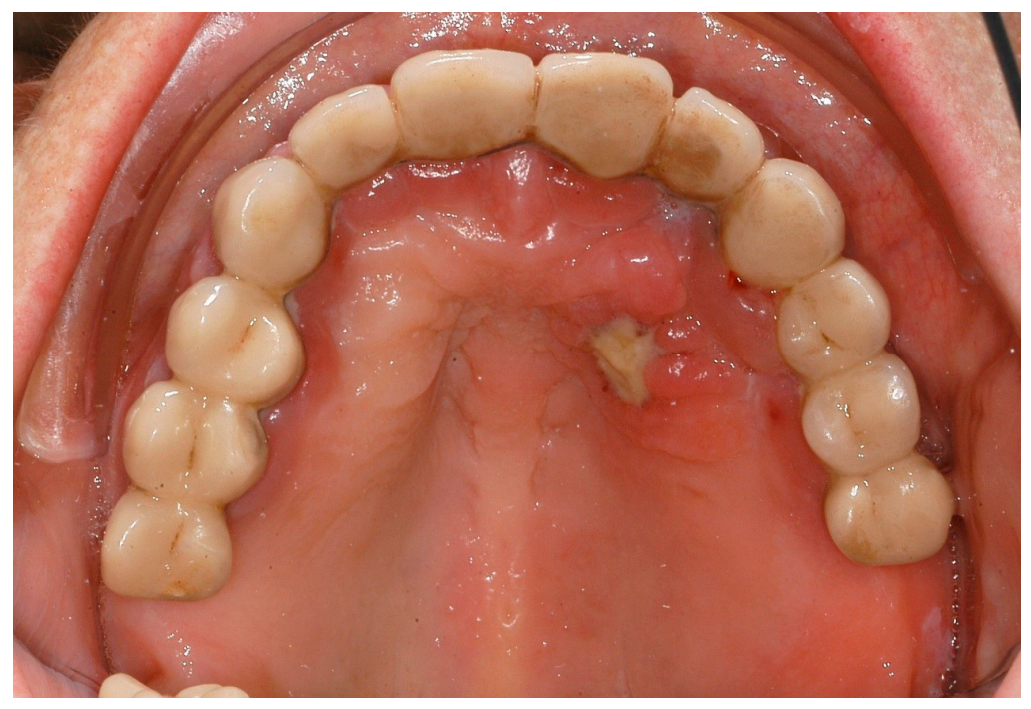

Figure 5. Gingival enlargement and necrosis as a sign of leukaemia in a 75-year-old women.

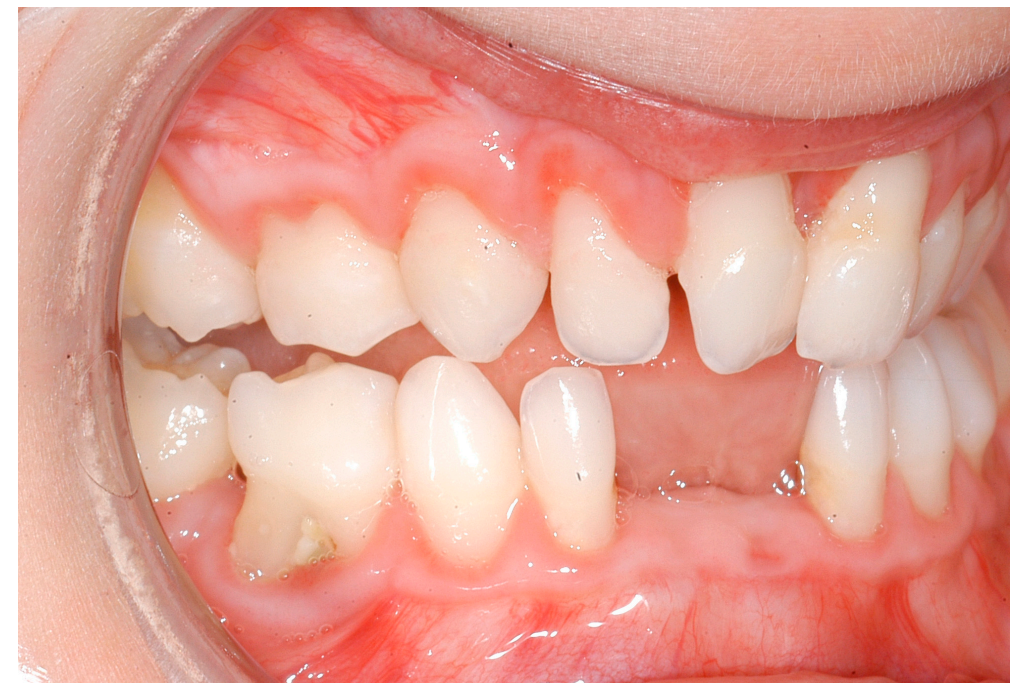

Figure 6. Five-year-old girl with Ehlers-Danlos Syndrome and periodontal destruction. 
Other available reports demonstrate that many oral health problems, as periodontal diseases and lower saliva flow rates, were found in subjects with neurodegenerative disease when compared with same age healthy individuals [39].

Hence, the professional must be aware of these aspects in order to diagnose periodontal disease correctly and to treat the patient accordingly.

This highlights the great importance of dental expertise in any early diagnosis. The list of RDs with periodontal manifestations in Table 1 should thus assist with the consideration of possible differential diagnoses in the event of there being a manifestation of unclear oral findings or observations that cannot be classified. Further information can then be retrieved from sources such as OMIM, Orphanet, or Pubmed that are stored in the database.

Classification systems are necessary as they provide a framework for scientific studies relating to aetiology, pathogenesis, and therapies [32]. To our knowledge, this study presents the first publication reporting RDs and their periodontal relevant manifestations with the view of facilitating the allocation of oral symptoms to a rare disease or of an oral disease to the oral symptoms as seen in clinical everyday life. Our work might thus extend the informative value of the new Classification of Periodontal and Peri-Implant Diseases and Conditions, which does not mention each of the specific rare diseases. An international network should now be developed, such as that for some RDs in the EU in the form of the European Reference Network [6], to enable the oral manifestation of RDs to be recorded in a targeted manner in the future. Dental medicine has not been included in this network to date.

Table 1. Rare diseases with periodontal manifestations: symptoms, pathological mechanism, affected gene, and classification according the 2018 version of the Classification of Periodontal and Peri-Implant Diseases and Conditions.

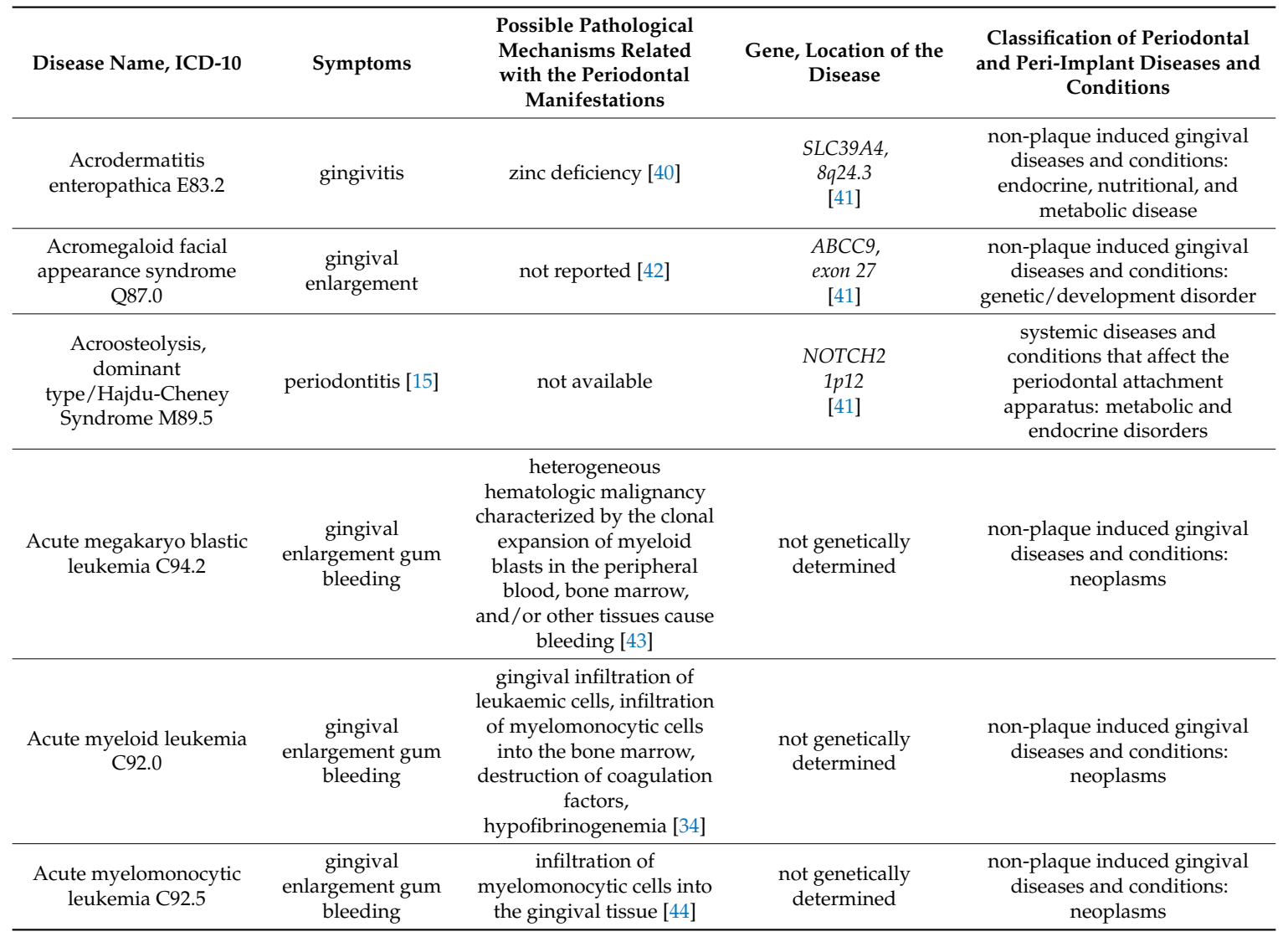


Table 1. Cont

\begin{tabular}{|c|c|c|c|c|}
\hline Disease Name, ICD-10 & Symptoms & $\begin{array}{l}\text { Possible Pathological } \\
\text { Mechanisms Related } \\
\text { with the Periodontal } \\
\text { Manifestations }\end{array}$ & $\begin{array}{l}\text { Gene, Location of the } \\
\text { Disease }\end{array}$ & $\begin{array}{l}\text { Classification of Periodontal } \\
\text { and Peri-Implant Diseases and } \\
\text { Conditions }\end{array}$ \\
\hline $\begin{array}{l}\text { Alopecia-epilepsy-pyorrhea- } \\
\text { intellectual disability } \\
\text { syndrome Q87.8 }\end{array}$ & gingivitis & unknown [45] & unknown [45] & $\begin{array}{c}\text { non-plaque induced gingival } \\
\text { diseases and conditions: } \\
\text { genetic/developmental } \\
\text { disorders }\end{array}$ \\
\hline $\begin{array}{c}\text { Amelogenesis } \\
\text { imperfecta-gingival } \\
\text { hyperplasia syndrome } \\
\text { K00.5 }\end{array}$ & $\begin{array}{l}\text { gingival } \\
\text { enlargement }\end{array}$ & $\begin{array}{l}\text { exact pathomechanism } \\
\text { unknown [46] }\end{array}$ & $\begin{array}{c}F A M 20 A, 17 \\
q 24.2[41]\end{array}$ & $\begin{array}{l}\text { non-plaque induced gingival } \\
\text { diseases and conditions: } \\
\text { genetic/developmental } \\
\text { disorders }\end{array}$ \\
\hline $\begin{array}{l}\text { Autosomal dominant } \\
\text { severe congenital } \\
\text { neutropenia D70 }\end{array}$ & periodontitis & neutropenia [48] & $\begin{array}{c}\text { ELANE 19p13.3 } \\
{[41]}\end{array}$ & $\begin{array}{l}\text { systemic diseases and } \\
\text { conditions that affect the } \\
\text { periodontal attachment } \\
\text { apparatus: diseases associated } \\
\text { with immunologic disorders }\end{array}$ \\
\hline $\begin{array}{c}\text { Bernard Soulier } \\
\text { syndrome/Giant platelet } \\
\text { syndrome D69.1 }\end{array}$ & $\begin{array}{l}\text { gum bleeding } \\
\text { bleeding diathesis }\end{array}$ & $\begin{array}{c}\text { low platelet count and } \\
\text { macrothrombocytopenia } \\
{[49]}\end{array}$ & $\begin{array}{c}\text { GP1BA,17p } \\
13.2[41]\end{array}$ & $\begin{array}{c}\text { non-plaque induced gingival } \\
\text { diseases and conditions: } \\
\text { genetic/developmental } \\
\text { disorders }\end{array}$ \\
\hline $\begin{array}{l}\text { Chediak-Higashi } \\
\text { Syndrom E70.3 }\end{array}$ & $\begin{array}{l}\text { gingivitis, } \\
\text { periodontits }\end{array}$ & $\begin{array}{c}\text { genetic disorder of granule } \\
\text { morphology and function } \\
{[50]}\end{array}$ & $\begin{array}{c}\operatorname{LYST} 1 q 42.3 \\
{[41]}\end{array}$ & $\begin{array}{l}\text { systemic diseases and } \\
\text { conditions that affect the } \\
\text { periodontal attachment } \\
\text { apparatus: diseases associated } \\
\text { with immunologic disorders }\end{array}$ \\
\hline Cohen syndrome Q87.8 & periodontitis & neutropenia [33] & $\begin{array}{l}\operatorname{VPS} 13 B, 8 q \\
22.2[41]\end{array}$ & $\begin{array}{l}\text { systemic diseases and } \\
\text { conditions that affect the } \\
\text { periodontal attachment } \\
\text { apparatus: diseases associated } \\
\text { with immunologic disorders }\end{array}$ \\
\hline $\begin{array}{l}\text { Congenital factor II } \\
\text { deficiency D68.2 }\end{array}$ & $\begin{array}{l}\text { gum bleeding } \\
\text { bleeding diathesis }\end{array}$ & $\begin{array}{c}\text { low prothrombin antigen } \\
\text { levels [52] }\end{array}$ & $\begin{array}{c}F 2,11 p 11.2 \\
{[41]}\end{array}$ & $\begin{array}{c}\text { non-plaque induced gingival } \\
\text { diseases and conditions: } \\
\text { genetic/developmental } \\
\text { disorders }\end{array}$ \\
\hline $\begin{array}{l}\text { Congenital factor V } \\
\text { deficiency D68.2 }\end{array}$ & $\begin{array}{l}\text { gum bleeding } \\
\text { bleeding diathesis }\end{array}$ & $\begin{array}{l}\text { low blood coagulation } \\
\text { factor V levels [ } 53]\end{array}$ & $\begin{array}{l}F 5,1 q 24.2 \\
\quad[41]\end{array}$ & $\begin{array}{c}\text { non-plaque induced gingival } \\
\text { diseases and conditions: } \\
\text { genetic/developmental } \\
\text { disorders }\end{array}$ \\
\hline $\begin{array}{l}\text { Congenital factor VII } \\
\text { deficiency D68.2 }\end{array}$ & $\begin{array}{l}\text { gum bleeding } \\
\text { bleeding diathesis }\end{array}$ & $\begin{array}{l}\text { low blood coagulation } \\
\text { factor VII levels [54] }\end{array}$ & $\begin{array}{l}F 7,13 q 34 \\
\quad[41]\end{array}$ & $\begin{array}{c}\text { non-plaque induced gingival } \\
\text { diseases and conditions: } \\
\text { genetic/developmental } \\
\text { disorders }\end{array}$ \\
\hline $\begin{array}{l}\text { Congenital factor X } \\
\text { deficiency D68.2 }\end{array}$ & $\begin{array}{l}\text { gum bleeding } \\
\text { bleeding diathesis }\end{array}$ & Factor X deficiency [55] & $\begin{array}{c}F 10,13 q 34 \\
{[41]}\end{array}$ & $\begin{array}{c}\text { non-plaque induced gingival } \\
\text { diseases and conditions: } \\
\text { genetic/developmental } \\
\text { disorders }\end{array}$ \\
\hline $\begin{array}{l}\text { Congenital factor XI } \\
\text { deficiency D68.1 }\end{array}$ & $\begin{array}{l}\text { gum bleeding } \\
\text { bleeding diathesis }\end{array}$ & Factor XI deficiency [56] & $\begin{array}{c}F 11,4 q 35.2 \\
{[41]}\end{array}$ & $\begin{array}{c}\text { non-plaque induced gingival } \\
\text { diseases and conditions: } \\
\text { genetic/developmental } \\
\text { disorders }\end{array}$ \\
\hline
\end{tabular}


Table 1. Cont.

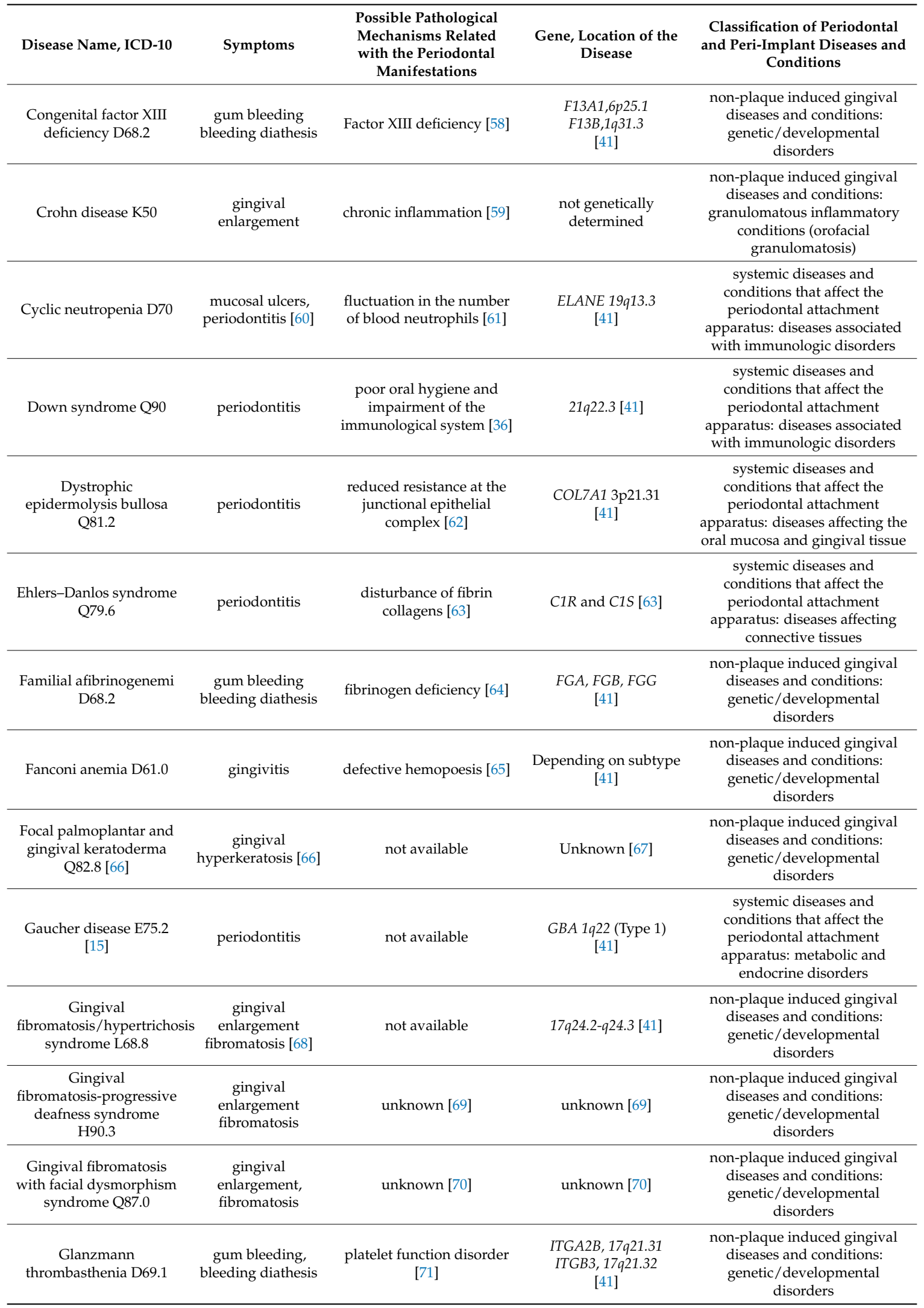


Table 1. Cont.

\begin{tabular}{|c|c|c|c|c|}
\hline Disease Name, ICD-10 & Symptoms & $\begin{array}{l}\text { Possible Pathological } \\
\text { Mechanisms Related } \\
\text { with the Periodontal } \\
\text { Manifestations }\end{array}$ & $\begin{array}{l}\text { Gene, Location of the } \\
\text { Disease }\end{array}$ & $\begin{array}{c}\text { Classification of Periodontal } \\
\text { and Peri-Implant Diseases and } \\
\text { Conditions }\end{array}$ \\
\hline $\begin{array}{l}\text { Glycogen storage disease } \\
1 \mathrm{~b} \text { E74.0 }\end{array}$ & $\begin{array}{l}\text { periodontal } \\
\text { breakdown }\end{array}$ & $\begin{array}{l}\text { neutrophil dysfunction } \\
\text { because of endoplasmic } \\
\text { retic-ulum stress generated } \\
\text { by disruption of } \\
\text { endogenous glucose } \\
\text { production [33] }\end{array}$ & $\begin{array}{c}\text { SLC37A4 11q23.3 } \\
{[41]}\end{array}$ & $\begin{array}{l}\text { systemic diseases and } \\
\text { conditions that affect the } \\
\text { periodontal attachment } \\
\text { apparatus: metabolic and } \\
\text { endocrine disorders }\end{array}$ \\
\hline $\begin{array}{l}\text { Granulomatosis with } \\
\text { polyangiitis M31.3 }\end{array}$ & $\begin{array}{l}\text { gingival } \\
\text { enlargement, } \\
\text { periodontitis }\end{array}$ & $\begin{array}{c}\text { production of } \\
\text { inflammatory cytocines } \\
{[13]}\end{array}$ & $\begin{array}{l}\text { not genetically } \\
\text { determined }\end{array}$ & $\begin{array}{l}\text { non-plaque induced gingival } \\
\text { diseases and conditions: } \\
\text { granulomatous inflammatory } \\
\text { conditions (orofacial } \\
\text { granulomatosis) systemic } \\
\text { diseases and conditions that } \\
\text { affect the periodontal } \\
\text { attachment apparatus: other } \\
\text { disorders that may affect } \\
\text { periodontal tissue }\end{array}$ \\
\hline $\begin{array}{l}\text { Hereditary gingival } \\
\text { fibromatosis K06.1 }\end{array}$ & $\begin{array}{l}\text { gingival } \\
\text { enlargement } \\
\text { fibromatosis }\end{array}$ & unknown [72] & $\begin{array}{c}\text { SOS1, 2p22.1 (Type 1) } \\
5 q 13-q 22 \text { (Type 2) } \\
2 p 23.3-p 22.3 \text { (Type } 3) \\
\text { 11p15 (Type 4) } \\
\text { REST, 4q12 (Type 5) } \\
{[41]}\end{array}$ & $\begin{array}{c}\text { non-plaque induced gingival } \\
\text { diseases and conditions: } \\
\text { genetic/developmental } \\
\text { disorders }\end{array}$ \\
\hline $\begin{array}{c}\text { Haim-Munk } \\
\text { syndrome/Keratosis } \\
\text { palmoplantaris- } \\
\text { periodontopathia- } \\
\text { onychogry-posis } \\
\text { syndrome Q82.8 }\end{array}$ & periodontitis & $\begin{array}{l}\text { exact pathomechanism } \\
\text { unknown [73] }\end{array}$ & $\begin{array}{l}\text { CTSC, } \\
11 q 14.2 \\
{[38,74]}\end{array}$ & $\begin{array}{l}\text { systemic diseases and } \\
\text { conditions that affect the } \\
\text { periodontal attachment } \\
\text { apparatus: diseases associated } \\
\text { with immunologic disorders }\end{array}$ \\
\hline Hemophilia A D66 & $\begin{array}{l}\text { gum bleeding } \\
\text { bleeding diathesis }\end{array}$ & Factor VIII deficiency [75] & $\begin{array}{c}F 8, X q 28 \\
{[41]}\end{array}$ & $\begin{array}{l}\text { non-plaque induced gingival } \\
\text { diseases and conditions: } \\
\text { genetic/developmental } \\
\text { disorders }\end{array}$ \\
\hline HemophiliaB D67 & $\begin{array}{l}\text { gum bleeding } \\
\text { bleeding diathesis }\end{array}$ & Factor IX deficiency [75] & $\begin{array}{c}F 9, X q 27.1 \\
{[41]}\end{array}$ & $\begin{array}{l}\text { non-plaque induced gingival } \\
\text { diseases and conditions: } \\
\text { genetic/developmental } \\
\text { disorders }\end{array}$ \\
\hline $\begin{array}{c}\text { Hennekam } \\
\text { syndrome/Lymphedema- } \\
\text { lymphangiectasia- } \\
\text { intellectual disability } \\
\text { syndrome Q87.8 }\end{array}$ & $\begin{array}{l}\text { gingival } \\
\text { enlargement }\end{array}$ & unknown [76] & $\begin{array}{c}\text { CCBE1,18q21.32 (Type } \\
\text { 1) } \\
\text { FAT4,4q28. (Type 2) } \\
{[41]}\end{array}$ & $\begin{array}{l}\text { non-plaque induced gingival } \\
\text { diseases and conditions: } \\
\text { genetic/developmental } \\
\text { disorders }\end{array}$ \\
\hline $\begin{array}{c}\text { Hereditary angioedema } \\
\text { (C-1-inhibitor deficiency) } \\
\text { D84.1 }\end{array}$ & periodontitis & $\begin{array}{l}\text { deficiency or functional } \\
\text { alteration of the C1 } \\
\text { inhibitor [77] }\end{array}$ & $\begin{array}{c}\text { C1NH 11q12.1 (Type 2) } \\
\text { F12 5q35.3 } \\
{[41]}\end{array}$ & $\begin{array}{l}\text { systemic diseases and } \\
\text { conditions that affect the } \\
\text { periodontal attachment } \\
\text { apparatus: diseases affecting } \\
\text { connective tissues }\end{array}$ \\
\hline Histoplasmosis D39 & gingival candidosis & $\begin{array}{c}\text { compromised } \\
\text { immunological system [78] }\end{array}$ & $\begin{array}{l}\text { not genetically } \\
\text { determined }\end{array}$ & $\begin{array}{c}\text { non-plaque induced gingival } \\
\text { diseases and conditions: } \\
\text { genetic/developmental } \\
\text { disorders }\end{array}$ \\
\hline $\begin{array}{l}\text { Hyalinosis cutis et } \\
\text { mucosae E78.8 }\end{array}$ & $\begin{array}{c}\text { gingival } \\
\text { enlargement }\end{array}$ & unknown [79] & $\begin{array}{c}\text { ECM1, } 1 q 21.2 \\
{[41]}\end{array}$ & $\begin{array}{c}\text { non-plaque induced gingival } \\
\text { diseases and conditions: } \\
\text { genetic/developmental } \\
\text { disorders }\end{array}$ \\
\hline $\begin{array}{c}\text { Hyper-IgE syndrome } \\
\text { D82.4 }\end{array}$ & $\begin{array}{l}\text { gingivitis, } \\
\text { periodontitis [15] }\end{array}$ & not available & $\begin{array}{c}\text { STAT3 17q21.2; DOCK8 } \\
9 q 24.3[41]\end{array}$ & $\begin{array}{l}\text { systemic diseases and } \\
\text { conditions that affect the } \\
\text { periodontal attachment } \\
\text { apparatus: diseases associated } \\
\text { with immunologic disorders }\end{array}$ \\
\hline $\begin{array}{l}\text { Hyper-IgM syndrome } \\
\text { with susceptibility to } \\
\text { opportunistic infections } \\
\text { D80.5 }\end{array}$ & periodontitis & $\begin{array}{c}\text { decreased levels of IgG } \\
\text { and IgA; elevated } \\
\text { concentrations ofIgM level } \\
{[80]}\end{array}$ & TNFSF5, Xq26.3 [41] & $\begin{array}{l}\text { systemic diseases and } \\
\text { conditions that affect the } \\
\text { periodontal attachment } \\
\text { apparatus: diseases associated } \\
\text { with immunologic disorders }\end{array}$ \\
\hline
\end{tabular}


Table 1. Cont

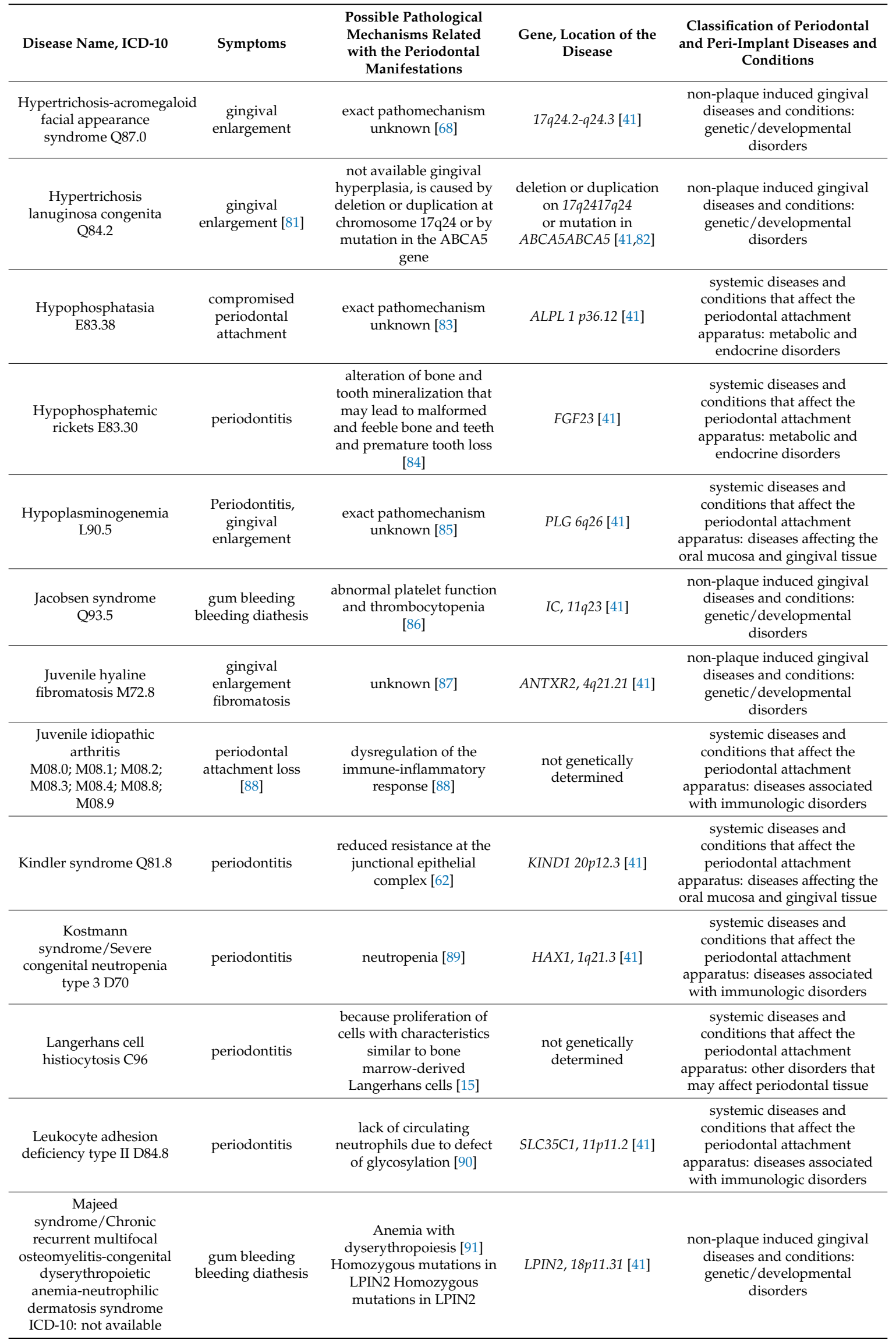


Table 1. Cont.

\begin{tabular}{|c|c|c|c|c|}
\hline Disease Name, ICD-10 & Symptoms & $\begin{array}{l}\text { Possible Pathological } \\
\text { Mechanisms Related } \\
\text { with the Periodontal } \\
\text { Manifestations }\end{array}$ & $\begin{array}{l}\text { Gene, Location of the } \\
\text { Disease }\end{array}$ & $\begin{array}{c}\text { Classification of Periodontal } \\
\text { and Peri-Implant Diseases and } \\
\text { Conditions }\end{array}$ \\
\hline $\begin{array}{c}\text { Papillon-Lefèvre } \\
\text { syndrome/Keratosis } \\
\text { palmoplantar- } \\
\text { periodontopathy } \\
\text { syndrome Q82.8 }\end{array}$ & periodontitis & $\begin{array}{l}\text { exact pathomechanism } \\
\text { unknown [72] }\end{array}$ & CTSC, $11 q 14.2[41,94]$ & $\begin{array}{l}\text { systemic diseases and } \\
\text { conditions that affect the } \\
\text { periodontal attachment } \\
\text { apparatus: diseases associated } \\
\text { with immunologic disorders }\end{array}$ \\
\hline $\begin{array}{c}\text { Ramon } \\
\text { syndrome/Cherubism- } \\
\text { gingival } \\
\text { fibromatosis-intellectual } \\
\text { disability syndrome } \\
\text { Q87.8 }\end{array}$ & $\begin{array}{l}\text { gingival } \\
\text { enlargement } \\
\text { fibromatosis }\end{array}$ & unknown [96] & $\begin{array}{l}\text { depending on subtype, } \\
\text { mostly unknown [96] }\end{array}$ & $\begin{array}{l}\text { non-plaque induced gingival } \\
\text { diseases and conditions: } \\
\text { genetic/developmental } \\
\text { disorders }\end{array}$ \\
\hline $\begin{array}{c}\text { Robinow } \\
\text { syndrome/Acral } \\
\text { dysostosis with facial } \\
\text { and genital } \\
\text { abnormalities Q87.1 }\end{array}$ & $\begin{array}{l}\text { gingival } \\
\text { enlargement }\end{array}$ & $\begin{array}{l}\text { mutant transcript in } \\
\text { fibroblasts [97] }\end{array}$ & $\begin{array}{c}\text { ROR2, 9q22.31 (Type: } \\
\text { autosomal-recessive) } \\
\text { WNT5A, 3p14.3 } \\
\text { (Type: dominant } 1 \text { ) } \\
\text { DVL1, } 1 p 36.33 \\
\text { (Type: dominant 2) } \\
\text { DVL3, 3q27.1 } \\
\text { (Type: dominant 3) } \\
\text { [41] }\end{array}$ & $\begin{array}{c}\text { non-plaque induced gingival } \\
\text { diseases and conditions: } \\
\text { genetic/developmental } \\
\text { disorders }\end{array}$ \\
\hline Seckel syndrome Q87.1 & $\begin{array}{c}\text { gingival } \\
\text { enlargement [99] }\end{array}$ & not available & $\begin{array}{c}\text { depending on subtype } \\
{[41]}\end{array}$ & $\begin{array}{c}\text { non-plaque induced gingival } \\
\text { diseases and conditions: } \\
\text { genetic/developmental } \\
\text { disorders }\end{array}$ \\
\hline $\begin{array}{c}\text { Segmental } \\
\text { odontomaxillary } \\
\text { dysplasia K00.4 }\end{array}$ & $\begin{array}{l}\text { gingival } \\
\text { enlargement }\end{array}$ & unknown [100] & $\begin{array}{l}\text { not genetically } \\
\text { determined }\end{array}$ & $\begin{array}{l}\text { non-plaque induced gingival } \\
\text { diseases and conditions: } \\
\text { genetic/developmental } \\
\text { disorders }\end{array}$ \\
\hline $\begin{array}{c}\text { Severe congenital } \\
\text { neutropenia D70 }\end{array}$ & periodontitis & neutropenia [48] & ELANE, $19 p 13.3$ [41] & $\begin{array}{l}\text { systemic diseases and } \\
\text { conditions that affect the } \\
\text { periodontal attachment } \\
\text { apparatus: diseases associated } \\
\text { with immunologic disorders }\end{array}$ \\
\hline $\begin{array}{c}\text { Systemic lupus } \\
\text { erythematosus M32 }\end{array}$ & $\begin{array}{l}\text { gingivitis, } \\
\text { periodontitis }\end{array}$ & $\begin{array}{l}\text { increased accumulation of } \\
\text { immune cells, } \\
\text { antineutrophil cytoplasm } \\
\text { antibodies and } \\
\text { metalloproteinases [101] }\end{array}$ & $\begin{array}{c}\text { PTPN22 1p13.2 } \\
\text { FCGR2A 1q23.3 } \\
\text { FCGR2B 1q23.3 } \\
\text { CTLA4 2q33.2 } \\
\text { TREX1 3p21.31 } \\
\text { DNASE1 16p13 } \\
{[41]} \\
\end{array}$ & $\begin{array}{l}\text { systemic diseases and } \\
\text { conditions that affect the } \\
\text { periodontal attachment } \\
\text { apparatus: diseases affecting } \\
\text { connective tissues }\end{array}$ \\
\hline
\end{tabular}


Table 1. Cont.

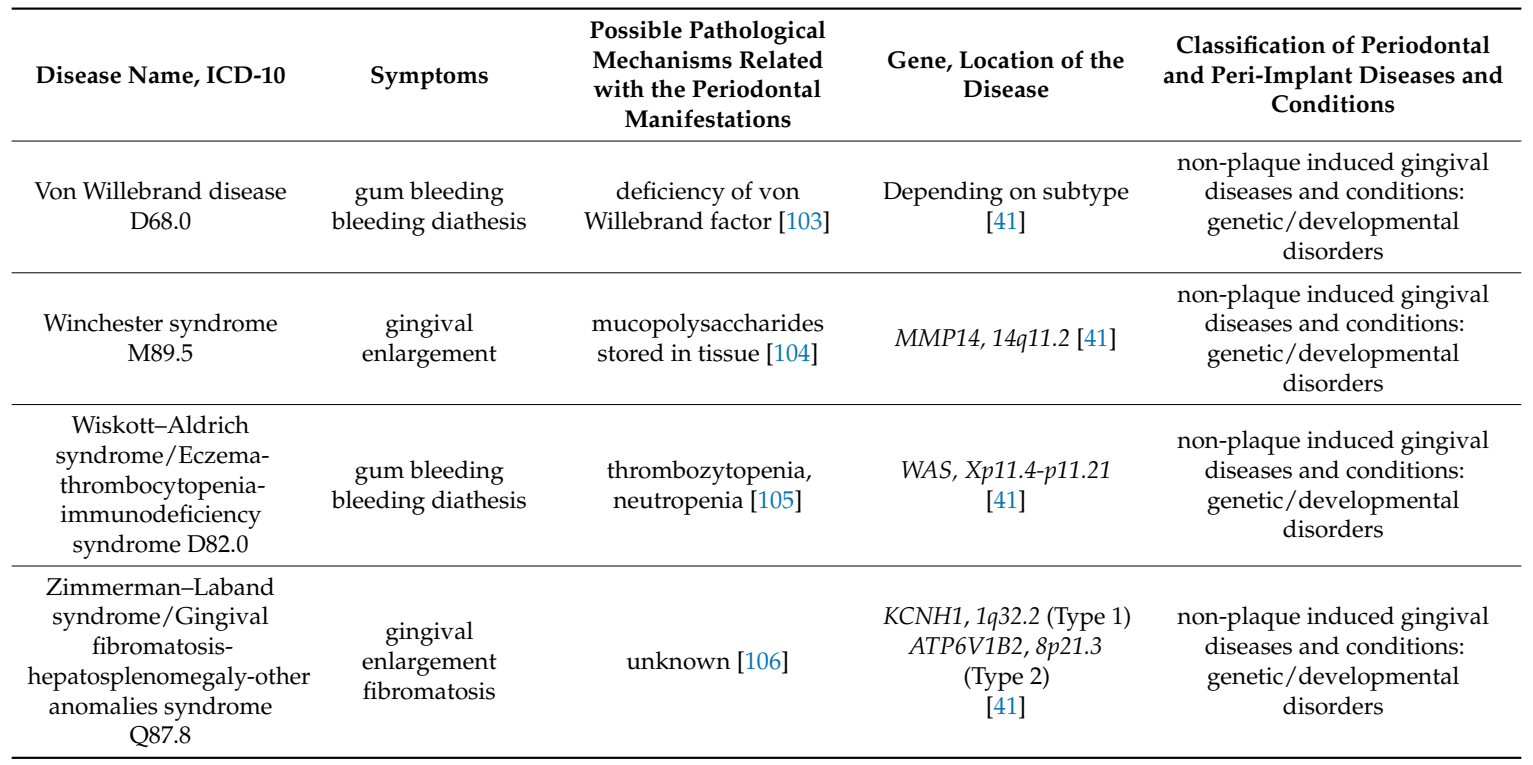

\section{Limitations of the Study}

The RDs analyzed in this study were described not by dentists, oral surgeons, or maxillofacial surgeons, but were based on cases in medical or genetic publications. The reason for this is the scant availability—or the complete lack of-dentistry publications.

Also, in the cases in which the study group made an individual assessment of the RD in question, based on the literature available, it is possible that some allocations of orofacial findings are wrong.

\section{Conclusions}

Approximately 14 percent of RDs with orofacial involvement also show manifestations in the form of gingivitis, periodontitis, and gingival hyperplasia in connection with the underlying disease. As a result, the dentist has an important role to play not only in the (accompanying) treatment, but also in any early diagnosis of the underlying disease, if he critically questions the symptoms, as described in this study, and can attribute them to a rare disease in individual cases. Also, the influence of these diseases on oral health and appropriate dental treatment to improve oral health should always be considered.

With the help of the Classification of Periodontal and Peri-Implant Diseases and Conditions, the scientific community is able to record RDs with periodontal manifestations more accurately in the future. This would mean that clinical data could be obtained more precisely to be able to assess the healthcare requirements for RDs.

In the future, there should be structured, standardized recordings of the symptoms of RDs, such as through a European alliance of university hospitals.

Author Contributions: Conception and Study design: M.H., T.H., L.H., and J.J.; Review of the Literature: L.B., M.H., K.B., and J.J.; J.K., and T.H. helped in the interpretation of data; M.H., L.H., and J.J. participated in design, and drafting of the manuscript. All authors read and approved the final manuscript.

Funding: This research received no external funding.

Conflicts of Interest: The authors declare no conflicts of interest.

\section{References}

1. Regulation (EC) No 141/2000 of the European Parliament and of the Council of 16 December 1999 on Orphan Medical Products. 1999. Available online: http:/ / ec.europa.eu/health/files/eudralex/vol-1/reg_2000_141_ cons-2009-07/reg_2000_141_cons-2009-07_en.pdf (accessed on 21 July 2018). 
2. German Federal Ministry of Health. 2013. Available online: http://www.bmg.bund.de/praevention/ gesundheitsgefahren/seltene-erkrankungen.html (accessed on 18 June 2017).

3. Wetterauer, B.; Schuster, R. Rare diseases. Funding programs in Germany and Europe. Bundesgesund. Gesundheitsforsch. Gesundheitssch. 2008, 51, 519. [CrossRef] [PubMed]

4. The Council of the European Union. Council Recommendation of 8 June 2009 on an Action in the Field of Rare Diseases (2009/C 151/02). Available online: http:/ / eur-lex.europa.eu/LexUriServ.do?uri=OJ:C:2009: 151:0007:0010:EN:PDF (accessed on 22 July 2018).

5. National Plan of Action for People with Rare Diseases: Action Fields, Recommendations, Proposed Actions. German Federal Ministry of Health, German Federal Ministry of Education and Research, Alliance for Chronic Rare Diseases 2013. Available online: http:/ / www.namse.de/ english.html (accessed on 9 October 2017).

6. Héon-Klin, V. European Reference networks for rare diseases: What is the conceptual framework? Orphanet. J. Rare Dis. 2017. [CrossRef] [PubMed]

7. European Organisation for Rare Diseases (EURODIS). Understanding this Public Health Priority. 2005. Available online: http:/ /www.eurordis.org/sites/default/files/publications/princeps_document-EN.pdf (accessed on 2 August 2018).

8. Reimann, A.; Bend, J.; Dembski, B. Patient-centred care in rare diseases. A patient organisations' perspective. Bundesgesund. Gesundheitsforsch. Gesundheitssch. 2007, 50, 1484. [CrossRef] [PubMed]

9. Rare Disease Impact Report: Insights from patients and the medical community. Shire 2013. Available online: https://globalgenes.org/wp-content/uploads/2013/04/ShireReport-1.pdf (accessed on 25 October 2018).

10. Kole, A.; Faurisson, F. Rare diseases social epidemiology: Analysis of inequalities. Adv. Exp. Med. Biol. 2010, 686, 223-250. [CrossRef] [PubMed]

11. Jackowski, J.; Hanisch, M. Orofacial manifestations in 2006 rare diseases-A preliminary systematic review of the literature. J. German Soc. Dent. Oral Med. 2012, 67, D10.

12. Hennekam, R.C.M.; Allanson, J.E.; Krantz, I.D.; Gorlin, R.J. Gorlin's Syndromes of the Head and Neck, 5th ed.; Oxford University Press: Oxford, UK, 2010.

13. Hanisch, M.; Fröhlich, L.F.; Kleinheinz, J. Gingival hyperplasia as first sign of recurrence of granulomatosis with polyangiitis (Wegener's granulomatosis): Case report and review of the literature. BMC Oral Health 2016, 17, 33. [CrossRef] [PubMed]

14. Eidt, D.; Frank, M.; Reimann, A.; Wagner, T.O.F.; Mittendorf, T.; Graf von der Schulenburg, J.M. Maßnahmen zur Verbesserung der Gesundheitlichen Situation von Menschen mit Seltenen Erkrankungen in Deutschland: Studie im Auftrag des Bundesministeriums für Gesundheit. Available online: https://www.bundesgesundheitsministerium.de/fileadmin/Dateien/5_Publikationen/Praevention/ Berichte/110516_Forschungsbericht_Seltene_Krankheiten.pdf (accessed on 28 October 2017).

15. Albandar, J.M.; Susin, C.; Hughes, F.J. Manifestations of systemic diseases and conditions that affect the periodontal attachment apparatus: Case definitions and diagnostic considerations. J. Periodontol. 2018, 89, S183-S203. [CrossRef]

16. Berglundh, T.; Armitage, G.; Araujo, M.G.; Avila-Ortiz, G.; Blanco, J.; Camargo, P.M.; Chen, S.; Cochran, D.; Derks, J.; Figuero, E.; Hämmerle, C.H.F.; et al. Peri-implant diseases and conditions: Consensus report of workgroup 4 of the 2017 World Workshop on the Classification of Periodontal and Peri-Implant Diseases and Conditions. J. Clin. Periodontol. 2018, 45, S286-S291. [CrossRef]

17. Caton, J.; Armitage, G.; Berglundh, T.; Chapple, I.L.C.; Jepsen, S.; Kornman, K.L.; Mealey, B.; Papapanou, P.N.; Sanz, M.; Tonetti, M.S. A new classification scheme for periodontal and peri-implant diseases and conditions-Introduction and key changes from the 1999 classification. J. Clin. Periodontol. 2018, 45, S1-S8. [CrossRef]

18. Chapple, I.L.C.; Mealey, B.L.; Van Dyke, T.E.; Bartold, P.M.; Dommisch, H.; Eickholz, P.; Geisinger, M.L.; Genco, R.J.; Glogauer, M.; Goldstein, M.; et al. Periodontal health and gingival diseases and conditions on an intact and a reduced periodontium: Consensus report of workgroup 1 of the 2017 World Workshop on the Classification of Periodontal and Peri-Implant Diseases and Conditions. J. Clin. Periodontol. 2018, 45, S68-S77. [CrossRef]

19. Cortellini, P.; Bissada, N.F. Mucogingival conditions in the natural dentition: Narrative review, case definitions, and diagnostic considerations. J. Clin. Periodontol. 2018, 45, S190-S198. [CrossRef] [PubMed] 
20. Herrera, D.; Retamal-Valdes, B.; Alonso, B.; Feres, M. Acute periodontal lesions (periodontal abscesses and necrotizing periodontal diseases) and endo-periodontal lesions. J. Clin. Periodontol. 2018, 45, S78-S94. [CrossRef] [PubMed]

21. Holmstrup, P.; Plemons, J.; Meyle, J. Non-plaque-induced gingival diseases. J. Clin. Periodontol. 2018, 45, S28-S43. [CrossRef] [PubMed]

22. Jepsen, S.; Caton, J.G.; Albandar, J.M.; Bissada, N.F.; Bouchard, P.; Cortellini, P.; Demirel, K.; de Sanctis, M.; Ercoli, C.; Fan, J.; et al. Periodontal manifestations of systemic diseases and developmental and acquired conditions: Consensus report of workgroup 3 of the 2017 World Workshop on the Classification of Periodontal and Peri-Implant Diseases and Conditions. J. Clin. Periodontol. 2018, 45, S219-S229. [CrossRef] [PubMed]

23. Papapanou, P.N.; Sanz, M.; Buduneli, N.; Dietrich, T.; Feres, M.; Fine, D.H.; Flemmig, T.F.; Garcia, R.; Giannobile, W.V.; Graziani, F.; Greenwell, H.; et al. Periodontitis: Consensus report of workgroup 2 of the 2017 World Workshop on the Classification of Periodontal and Peri-Implant Diseases and Conditions. J. Clin. Periodontol. 2018, 45, S162-S170. [CrossRef] [PubMed]

24. Tonetti, M.S.; Greenwell, H.; Kornman, K.S. Staging and grading of periodontitis: Framework and proposal of a new classification and case definition. J. Clin. Periodontol. 2018, 45, S149-S161. [CrossRef]

25. ROMSE. Available online: http:/ / romse.org (accessed on 2 January 2019).

26. Hanisch, M.; Hanisch, L.; Benz, K.; Kleinheinz, J.; Jackowski, J. Development of a database to record orofacial manifestations in patients with rare diseases: A status report from the ROMSE (recording of orofacial manifestations in people with rare diseases) database. Br. J. Oral Maxillofac. Surg. 2017, 55, 500-503. [CrossRef]

27. List of Rare Diseases and Synonyms in Alphabetical Order. May 2015 Version. Available online: http:/ / www. orpha.net/consor4.01/www/cgi-bin/Education_Home.php?lng=DE\#REPORT_RARE_DISEASES (accessed on 8 May 2015).

28. PubMed. Available online: https://www.ncbi.nlm.nih.gov (accessed on 6 October 2018).

29. Müller, A. Auflistung aller Parodontal-Relevanten Seltenen Erkrankungen mit den Anfangsbuchstaben A-K. Ph.D. Thesis, Dresden, Germany, 2017.

30. Molina-García, A.; Castellanos-Cosano, L.; Machuca-Portillo, G.; Posada-de la Paz, M. Impact of rare diseases in oral health. Med. Oral Patol. Oral Cir. Bucal. 2016, 21, e587-e594.

31. Corcuera-Flores, J.R.; Casttellanos-Cosano, L.; Torres-Lagares, D.; Serrera-Figallo, M.Á.; Rodríguez-Caballero, Á.; Machuca-Portillo, G. A systematic review of the oral and craniofacial manifestations of cri of the cat syndrome. Clin Anat. 2016, 29, 29-555. [CrossRef]

32. Armitage, G.C. Development of classification system for periodontal diseases and conditions. Ann. Periodontol. 1999, 4, 1-6. [CrossRef]

33. Khocht, A.; Albandar, J.M. Aggressive forms of periodontitis secondary to systemic disorders. Periodontology 2000, 65, 134-148. [CrossRef] [PubMed]

34. Guan, G.; Firth, N. Oral manifestations as an early clinical sign of acute myeloid leukaemia: A case report. Aust. Dent. J. 2015, 60, 123-127. [CrossRef]

35. Matsuo, F.S.; Barbosa de Paulo, L.F.; Servato, J.P.; de Faria, P.R.; Cardoso, S.V.; Loyola, A.M. Involvement of oral tissues by AL amyloidosis: A literature review and report of eight new cases. Clin. Oral Investig. 2016, 20, 1913-1920. [CrossRef] [PubMed]

36. Ferreira, R.; Michel, R.C.; Greghi, S.L.; Resende, M.L.; Sant'Ana, A.C.; Damante, C.A. Prevention and Periodontal Treatment in Down Syndrome Patients: A Systematic Review. PLoS ONE 2016, 11, e0158339. [CrossRef] [PubMed]

37. Hanisch, M.; Jung, S.; Kleinheinz, J. Identification of rare diseases in the oral cavity. Internist 2018, 59, 972-980. [CrossRef] [PubMed]

38. Berglund, B.; Björck, K. Women with Ehlers-Danlos Syndrome experience low oral health-related quality of life. J. Orofac. Pain 2012, 26, 307-314. [PubMed]

39. Cicciù, M. Neurodegenerative Disorders and Periodontal Disease: Is There a Logical Connection? Neuroepidemiology 2016, 47, 94-95. [CrossRef] [PubMed]

40. Dev, T.; Sethuraman, G. Diagnosis of acrodermatitis enteropathica in resource limited settings. BMJ Case Rep. 2017. [CrossRef]

41. OMIM: Online Inheritance in Man. Available online: http:/ / www.omim.org (accessed on 6 October 2018). 
42. Afifi, H.H.; Abdel-Hamid, M.S.; Eid, M.M.; Mostafa, I.S.; Abdel-Salam, G.M. De Novo Mutation in ABCC9 Causes Hypertrichosis Acromegaloid Facial Features Disorder. Pediatr. Dermatol. 2016, 2, e109-e113. [CrossRef]

43. O’Donnell, M.R.; Tallman, M.S.; Abboud, C.N.; Altman, J.K.; Appelbaum, F.R.; Arber, D.A.; Bhatt, V.; Bixby, D.; Blum, W.; Coutre, S.E.; et al. Acute Myeloid Leukemia, Version 3.2017, NCCN Clinical Practice Guidelines in Oncology. J. Natl. Compr. Canc. Netw. 2017, 15, 926-957. [CrossRef]

44. Sonoi, N.; Soga, Y.; Maeda, H.; Ichimura, K.; Yoshino, T.; Aoyama, K. Histological and immunohistochemical features of gingival enlargement in a patient with AML. Odontology 2012, 100, 254-257. [CrossRef] [PubMed]

45. Kini, U.; Clayton-Smith, J. Acromegaloid facial appearance syndrome: A further case report. Clin. Dysmorphol. 2004, 13, 251-253. [CrossRef] [PubMed]

46. Li, L.L.; Liu, P.H.; Xie, X.H.; Ma, S.; Liu, C.; Chen, L. Loss of epithelial FAM20A in mice causes amelogenesis imperfecta, tooth eruption delay and gingival overgrowth. Int. J. Oral Sci. 2016, 8, 98-109. [CrossRef] [PubMed]

47. Venclikova, Z.; Benada, O.; Joska, L. Monitoring of selenium in oral cavity argyria-A clinical and microscopic study. Neuro Endocrinol. Lett. 2011, 32, 286-291. [PubMed]

48. Donadieu, J.; Fenneteau, O.; Beaupain, B.; Mahlaoui, N.; Chantelot, C.B. Congenital neutropenia: Diagnosis, molecular bases and patient management. Orphanet. J. Rare Dis. 2011, 6, 26. [CrossRef] [PubMed]

49. Hartman, M.J.; Caccamese, J.F., Jr.; Bergman, S.A. Perioperative management of a patient with Bernard-Soulier syndrome for third molar surgery. Oral Surg. Oral Med. Oral Pathol. Oral Radiol. Endod 2007, 103, 626-629. [CrossRef] [PubMed]

50. Ward, D.M.; Shiflett, S.L.; Kaplan, J. Chediak-Higashi syndrome: A clinical and molecular view of a rare lysosomal storage disorder. Curr. Mol. Med. 2002, 2, 469-477. [CrossRef] [PubMed]

51. Buduneli, N.; Baylas, H.; Aksu, G.; Kutukculer, N. Prepubertal periodontitis associated with chronic granulomatous disease. J. Clin. Periodontol. 2001, 28, 589-593. [CrossRef]

52. Girolami, A.; Santarossa, C.; Cosi, E.; Ferrari, S.; Lombardi, A.M.; Girolami, B. Bleeding manifestations in heterozygotes with prothrombin deficiency or abnormalities vs. unaffected family members as observed during a long follow-up study. Blood Coagul. Fibrinolysis 2017, 28, 623-626. [CrossRef]

53. Naderi, M.; Tabibian, S.; Alizadeh, S.; Hosseini, S.; Zaker, F.; Bamedi, T. Congenital factor V deficiency: Comparison of the severity of clinical presentations among patients with rare bleeding disorders. Acta Haematol. 2015, 133, 148-154. [CrossRef]

54. Weinstock, R.J.; Onyejiuwa, A.; Shnayder, G.; Clarkson, E.I. Use of recombinant factor VII for tooth extractions in a patient with severe congenital factor VII deficiency: A case report. J. Am. Dent. Assoc. 2015, 146, 271-275. [CrossRef] [PubMed]

55. Menegatti, M.; Peyvandi, F. Factor X deficiency. Semin. Thromb. Hemost. 2009, 35, 407-415. [CrossRef] [PubMed]

56. Bolton-Maggs, P.H. Factor XI deficiency and its management. Haemophilia 2000, 6, 100-109. [CrossRef] [PubMed]

57. Bender, L.; Weidmann, H.; Rose-John, S.; Renne, T.; Long, A.T. Factor XII-Driven Inflammatory Reactions with Implications for Anaphylaxis. Front Immunol. 2017, 8, 1115. [CrossRef] [PubMed]

58. Karimi, M.; Cairo, A.; Safarpour, M.M.; Haghpanah, S.; Ekramzadeh, M.; Afrasiabi, A. Genotype and phenotype report on patients with combined deficiency of factor V and factor VIII in Iran. Blood Coagul. Fibrinolysis 2014, 25, 360-363. [CrossRef] [PubMed]

59. Scheper, H.J.; Brand, H.S. Oral aspects of Crohn's disease. Int. Dent. J. 2002, 52, 163-172. [CrossRef] [PubMed]

60. Chen, Y.; Fang, L.; Yang, X. Cyclic neutropenia presenting as recurrent oral ulcers and periodontitis. J. Clin. Pediatr. Dent. 2013, 37, 307-308. [CrossRef] [PubMed]

61. Migliaccio, A.R.; Migliaccio, G.; Dale, D.C.; Hammond, W.P. Hematopoietic progenitors in cyclic neutropenia: Effect of granulocyte colony-stimulating factor in vivo. Blood 1990, 75, 1951-1959.

62. Wiebe, C.B.; Larjava, H.S. Do mutations in the basement membrane zone affect the human periodontium? Review with special reference to epidermolysis bullosa. J. West Soc. Periodontol. Periodontal. Abstr. 1998, 46, 5-18. [PubMed]

63. Kapferer-Seebacher, I.; Lundberg, P.; Malfait, F.; Zschocke, J. Periodontal manifestations of Ehlers-Danlos syndromes: A systematic review. J. Clin. Periodontol. 2017, 44, 1088-1100. [CrossRef] [PubMed] 
64. Naz, A.; Biswas, A.; Khan, T.N.; Goodeve, A.; Ahmed, N.; Saqlain, N. Identification of novel mutations in congenital afibrinogenemia patients and molecular modeling of missense mutations in Pakistani population. Thromb. J. 2017, 15, 24. [CrossRef] [PubMed]

65. Goswami, M.; Bhushan, U. Dental perspective of rare disease of Fanconi anemia: Case report with review. Clin. Med. Insights Case Rep. 2016, 17, 25-30. [CrossRef] [PubMed]

66. Gorlin, R.J. Focal palmoplantar and marginal gingival hyperkeratosis-A syndrome. Birth Defects Orig. Artic. Ser. 1976, 12, 239-242. [PubMed]

67. Bethke, G.; Kolde, G.; Bethke, G.; Reichart, P.A. Focal palmoplantar and oral mucosa hyperkeratosis syndrome. Mund. Kiefer Gesichtschir. 2001, 5, 202-205. [CrossRef] [PubMed]

68. Sun, M.; Li, N.; Dong, W.; Chen, Z.; Liu, Q.; Xu, Y.; He, G.; Shi, Y.; Li, X.; Hao, J.; et al. Copy-number mutations on chromosome 17q24.2-q24.3 in congenital generalized hypertrichosis terminalis with or without gingival hyperplasia. Am. J. Hum. Genet. 2009, 84, 807-813. [CrossRef] [PubMed]

69. Hartsfield, J.K., Jr.; Bixler, D.; Hazen, R.H. Gingival fibromatosis with sensorineural hearing loss: An autosomal dominant trait. Am. J. Med. Genet. 1985, 22, 623-627. [CrossRef]

70. Goldblatt, J.; Singer, S.L. Autosomal recessive gingival fibromatosis with distinctive facies. Clin. Genet. 1992, 42, 306-308. [CrossRef]

71. Toygar, H.U.; Guzeldemir, E. Excessive gingival bleeding in two patients with Glanzmann thrombasthenia. J. Periodontol. 2007, 78, 1154-1158. [CrossRef]

72. Xiao, S.; Bu, L.; Zhu, L.; Zheng, G.; Yang, M.; Qian, M.; Hu, L.; Liu, J.; Zhao, G.; Kong, X. A new locus for hereditary gingival fibromatosis (GINGF2) maps to 5q13-q22. Genomics 2001, 74, 180-185. [CrossRef]

73. Korkmaz, B.; Caughey, G.H.; Chapple, I.; Gauthier, F.; Hirschfeld, J.; Jenne, D.E.; Kettritz, R.; Lalmanach, G.; Lamort, A.S.; Lauritzen, C.; et al. Therapeutic targeting of cathepsin C: From pathophysiology to treatment. Pharmacol. Ther. 2018. [CrossRef]

74. Hart, T.C.; Hart, P.S.; Michalec, M.D.; Zhang, Y.; Firatli, E.; Van Dyke, T.E.; Stabholz, A.; Zlotogorski, A.; Shapira, L.; Soskolne, W.A. Haim-Munk syndrome and Papillon-Lefèvre syndrome are allelic mutations in cathepsin C. J. Med. Genet. 2000, 37, 88-94. [CrossRef]

75. Mannucci, P.M.; Tuddenham, E.G. The hemophilias-From royal genes to gene therapy. N. Engl. J. Med. 2001, 344, 1773-1779. [CrossRef] [PubMed]

76. Scarcella, A.; De Lucia, A.; Pasquariello, M.B.; Gambardella, P. Early death in two sisters with Hennekam syndrome. Am. J. Med. Genet. 2000, 93, 181-183. [CrossRef]

77. Davis, A.E. The pathophysiology of hereditary angioedema. Clin. Immunol. 2005, 114, 3-9. [CrossRef] [PubMed]

78. Kauffman, C.A. Histoplasmosis: A clinical and laboratory update. Clin. Microbiol. Rev. 2007, 20, 115-132. [CrossRef] [PubMed]

79. Xanthinaki, A.A.; Donta, C.; Gatou, V.; Tsichlakis, C. Hyalinosis cutis et mucosae: Diagnosis based on oral manifestations-Report of a case. J. Clin. Pediatr. Dent. 2008, 33, 171-174. [CrossRef] [PubMed]

80. Levy, J.; Espanol-Boren, T.; Thomas, C.; Fischer, A.; Tovo, P.; Bordigoni, P.; Resnick, I.; Fasth, A.; Baer, M.; Gomez, L.; et al. Clinical spectrum of X-linked hyper-IgM syndrome. J. Pediatr. 1997, 131, 47-54. [CrossRef]

81. Tadin-Strapps, M.; Salas-Alanis, J.C.; Moreno, L.; Warburton, D.; Martinez-Mir, A.; Christiano, A.M. Congenital universal hypertrichosis with deafness and dental anomalies inherited as an X-linked trait. Clin. Genet. 2003, 63, 418-422. [CrossRef]

82. Fantauzzo, K.A.; Kurban, M.; Levy, B.; Christiano, A.M. Trps1 and its target gene Sox9 regulate epithelial proliferation in the developing hair follicle and are associated with hypertrichosis. PLoS Genet. 2012, 8, e1003002. [CrossRef]

83. Foster, B.L.; Kuss, P.; Yadav, M.C.; Kolli, T.N.; Narisawa, S.; Lukashova, L.; Cory, E.; Sah, R.L.; Somerman, M.J.; Millán, J.L. Conditional Alpl Ablation Phenocopies Dental Defects of Hypophosphatasia. J. Dent. Res. 2017, 96, 81-91. [CrossRef]

84. McKee, M.D.; Hoac, B.; Addison, W.N.; Barros, N.M.; Millan, J.L.; Chaussain, C. Extracellular matrix mineralization in periodontal tissues: Noncollagenous matrix proteins, enzymes, and relationship to hypophosphatasia and X-linked hypophosphatemia. Periodontology 2000, 63, 102-122. [CrossRef] [PubMed]

85. Sulniute, R.; Lindh, T.; Wilczynska, M.; Li, J.; Ny, T. Plasmin is essential in preventing periodontitis in mice. Am. J. Pathol. 2011, 179, 819-828. [CrossRef] [PubMed] 
86. Ichimiya, Y.; Wada, Y.; Kunishima, S.; Tsukamoto, K.; Kosaki, R.; Sago, H.; Ishiguro, A.; Ito, Y. 11q23 deletion syndrome (Jacobsen syndrome) with severe bleeding: A case report. J. Med. Case Rep. 2018. [CrossRef] [PubMed]

87. Denadai, R.; Raposo-Amaral, C.E.; Bertola, D.; Kim, C.; Alonso, N.; Hart, T.; Han, S.; Stelini, R.F.; Buzzo, C.L.; Raposo-Amaral, C.A.; et al. Identification of 2 novel ANTXR2 mutations in patients with hyaline fibromatosis syndrome and proposal of a modified grading system. Am. J. Med. Genet. 2012, 158, 732-742. [CrossRef] [PubMed]

88. Miranda, L.A.; Fischer, R.G.; Sztajnbok, F.R.; Figueredo, C.M.; Gustafsson, A. Periodontal conditions in patients with juvenile idiopathic arthritis. J. Clin. Periodontol. 2003, 30, 969-974. [CrossRef] [PubMed]

89. Boztug, K.; Ding, X.-Q.; Hartmann, H.; Ziesenitz, L.; Schaffer, A.A.; Diestelhorst, J.; Pfeifer, D.; Appaswamy, G.; Kehbel, S.; Simon, T.; et al. HAX1 mutations causing severe congenital neuropenia (sic) and neurological disease lead to cerebral microstructural abnormalities documented by quantitative MRI. Am. J. Med. Genet. 2010, 152, 3157-3163. [CrossRef] [PubMed]

90. Hajishengallis, G.; Moutsopoulos, N.M.; Hajishengallis, E.; Chavakis, T. Immune and regulatory functions of neutrophils in inflammatory bone loss. Semin. Immunol. 2016, 28, 146-158. [CrossRef] [PubMed]

91. Ferguson, P.J.; Chen, S.; Tayeh, M.K.; Ochoa, L.; Leal, S.M.; Pelet, A.; Munnich, A.; Lyonnet, S.; Majeed, H.A.; El-Shanti, H. Homozygous mutations in LPIN2 are responsible for the syndrome of chronic recurrent multifocal osteomyelitis and congenital dyserythropoietic anaemia (Majeed syndrome). J. Med. Genet. 2005, 42, 551-557. [CrossRef]

92. Encarnacao, M.; Lacerda, L.; Costa, R.; Prata, M.J.; Coutinho, M.F.; Ribeiro, H.; Lopes, L.; Pineda, M.; Ignatius, J.; Galvez, H.; et al. Molecular analysis of the GNPTAB and GNPTG genes in 13 patients with mucolipidosis type II or type III-Identification of eight novel mutations. Clin. Genet. 2009, 76, 76-84. [CrossRef]

93. Tartaglia, M.; Kalidas, K.; Shaw, A.; Song, X.; Musat, D.L.; van der Burgt, I.; Brunner, H.G.; Bertola, D.R.; Crosby, A.; Ion, A.; et al. PTPN11 mutations in Noonan syndrome: Molecular spectrum, genotype-phenotype correlation, and phenotypic heterogeneity. Am. J. Hum. Genet. 2002, 70, 1555-1563. [CrossRef]

94. Moghaddasian, M.; Arab, H.; Dadkhah, E.; Boostani, H.; Babak, A.R.; Abbaszadegan, M.R. Protein modeling of cathepsin C mutations found in Papillon-Lefèvre syndrome. Gene 2014, 538, 182-187. [CrossRef] [PubMed]

95. McNamara, K.M.; Hall, S.E.; Wilder, R.S.; Lawrence, H.P.; Offenbacher, S. Periodontitis and cytokine expression in CD14 deficient patients. J. Int. Acad. Periodontol. 1999, 1, 95-100. [PubMed]

96. Gawron, K.; Łazarz-Bartyzel, K.; Potempa, J.; Chomyszyn-Gajewska, M. Gingival fibromatosis: Clinical, molecular and therapeutic issues. Orphanet. J. Rare Dis. 2016, 11, 9. [CrossRef] [PubMed]

97. Bunn, K.J.; Daniel, P.; Rösken, H.S.; O’Neill, A.C.; Cameron-Christie, S.R.; Morgan, T.; Brunner, H.G.; Lai, A.; Kunst, H.P.; Markie, D.M.; et al. Mutations in DVL1 cause an osteosclerotic form of Robinow syndrome. Am. J. Hum. Genet. 2015, 96, 623-630. [CrossRef] [PubMed]

98. Murai, O.; Naruishi, K.; Ogihara, S.; Suwa, N.; Kanazawa, S.; Yaegashi, T.; Takeda, Y.; Kunimatsu, K. Cathepsin $\mathrm{B}, \mathrm{D}$, and L regulation in cyclosporin A-mediated gingival hyperplasia of a patient with sarcoidosis. Clin. Lab. 2011, 57, 535-541. [PubMed]

99. Regen, A.; Nelson, L.P.; Woo, S.B. Dental manifestations associated with Seckel syndrome type II: A case report. Pediatr Dent. 2010, 32, 445-450. [PubMed]

100. Agrawal, A.; Daniel, J.; Vijeev, V. Segmental odontomaxillary dysplasia. J. Indian Soc. Pedod Prev. Dent. 2014, 32, 83-86. [CrossRef] [PubMed]

101. Marques, C.P.; Maor, Y.; de Andrade, M.S.; Rodrigues, V.P.; Benatti, B.B. Possible evidence of systemic lupus erythematosus and periodontal disease association mediated by Toll-like receptors 2 and 4. Clin. Exp. Immunol. 2016, 183, 187-192. [CrossRef]

102. Pischon, N.; Hoedke, D.; Kurth, S.; Lee, P.; Dommisch, H.; Steinbrecher, A.; Pischon, T.; Burmester, G.R.; Buttgereit, F.; Detert, J.; et al. Increased Periodontal Attachment Loss in Patients with Systemic Sclerosis. J. Periodontol. 2016, 87, 763-771. [CrossRef]

103. Castaman, G.; Linari, S. Diagnosis and Treatment of von Willebrand Disease and Rare Bleeding Disorders. J. Clin. Med. 2017. [CrossRef]

104. Prapanpoch, S.; Jorgenson, R.J.; Langlais, R.P.; Nummikoski, P.V. Winchester syndrome. A case report and literature review. Oral Surg. Oral Med. Oral Pathol. 1992, 74, 671-677. [CrossRef] 
105. Lemahieu, V.; Gastier, J.M.; Francke, U. Novel mutations in the Wiskott-Aldrich syndrome protein gene and their effects on transcriptional, translational, and clinical phenotypes. Hum. Mutat. 1999, 14, 54-66. [CrossRef]

106. Balasubramanian, M.; Parker, M.J. Zimmermann-Laband syndrome in a child previously described with brachydactyly, extrahepatic biliary atresia, patent ductus arteriosus and seizures. Clin. Dysmorph. 2010, 19, 48-50. [CrossRef] [PubMed] 